moment of time? If you have, you have surely caught yourself wishing that you could but have followed yourself on through that space-your body mute but the spirit untrammelled in the acuteness of its perceptions of that just beyond-for then would you not have known what death was, would you not have got a glimpse into that unknown land where time shall be no longer? It all seemed so disappointingly possible, and yet it was not. Perhaps wireless telegraphy in its suggestion of containing the germ of an explanation of the physical basis of thought transference is as disappointing. Who can tell ?

One other aspect of my subject occurs to me of even less practical bearing and yet it is one that $I$ would not leave untouched, for it is perhaps more at one with the spirit of the stanza that has served me for a text. That "tread softly" breathes a reverent attitude of mind; let it so stand towards the experience of which I have spoken. I was once told-and having been a somewhat copious note-taker of such cases as have come before me I heard it with dismay - that I should find my notes of little use to myself and that they would be of none at all to those who should come after me. And, alas, I believe that my friend had some reason for his opinion, for many a time $I$ have found a case to be full of interest at the moment of investigation and full of important suggestions on what seemed at the time to be promising lines of inquiry which, when I came back to it after a time, was absolutely lifeless. I could not record the spirit of the thing any more than the painter can depict the soul that animates the husk of man. You can record a unique case but you cannot record the points that are special to each one of a common group of cases. And you cannot because: firstly, they will probably strike you, if at all, only once and for a moment and no more; and then if you could they would convey little meaning to other minds, for these relationships and affinities between one disease and another, these flashes of thought direct from one thing to perhaps another that has no obvious feature in common, are of the nature of intuitions-inexplicable, perhaps not even true for the general, but instructive and very real for the individual ; and sometimes, no doubt, containing a suggestion, if it could be followed up, of possibly some important observation. And I suspect that when one talks of experience this is the essence of its value : a possession that, if time does not fail, may shoot upwards with ever-increasing insight and concentration of vision until the top of it reaches to heaven. But, alas, time does mostly fail, and potential fruition melts away, its promise unfulfilled. Is it not true that each one of the sages of medicine--and I count as such all those who have lived their, say, 60 years of busy and observant life-is filled with nascent knowledge of this kind and that as he passes into the unknown by far the larger part of his knowledge goes with him? And that "tread softly" does but express the intense longing everyone must feel that it were possible to distil the essence and to prevent the waste (is it waste?) of that memory that sleeps. And we may well remember that each one of us possesses an experience that is both individual and unique, and that there is not one of us that has not within him plenty of valuable material which if he could only render it up, might advance materially the general stock of knowledge, and accelerate the progress of medicine in a measure past our dreaming.

Where memory sleeps! Tread softly, tread reverently, tread inquiringly, beside this still mysterious attribute of life, and when, as it must and does, its silent influence stirs within these walls, built and furnished, as I understand, by the generosity of one who happily still lives amongst you, be sure of this : it blesses him that gave, the sick who are the recipients of skill and comfort they might not otherwise have obtained, and you who work herein.

Donations and Bequests.-By the will of Mr. Samuel Palmer of Northcourt, Hampstead, the North London Hospital for Consumption at Mount Vernon receives $£ 500$ and the St. John's Wood and Portland Town Provident Dispensary and the Royal Hospital for Incurables similar amounts. - Mr. John Wilder of Erleigh Grange, Reading, has by his will bequeathed $£ 500$ to the Royal Berkshire Hospital. - Mr. Richard Bishop of Acton has by his will left $£ 500$ to St. Thomas's Hospital. The residue of the estate, after the payment of certain personal bequests, is left upon trust for division nmmar $m$ mh hnspitals in the county of London as the traste may think fit.

\section{ON THE \\ TREATMENT OF BUBONIC PLAGUE BY YERSIN'S SERUM, WITH OBSER- VATIONS ON ITS MODE OF ACTION.}

By D. LOUIS CAIRNS, M.D. GlasG,

IATE SENIOR RESIDENT ASSISTANT PHYSICIAN, CITY OF GLASGOW FENF HOSPITAL, BELVIDERE, GLASGOW.

IN a previous communication to THE LANCET ${ }^{1}$ I described at some length investigations into the "agglutinative reaction in cases of bubonic plague. Whilst pursuing these experiments on agglutination other lines of research were opened up and I have thought that an account of these further investigations might be of interest to some of the readers of THE LANCET. Advantage was taken of the second outbreak of plague in 1901 to examine more fully certain morphological alterations which had been observed to take place in the bacillus pestis, both in the bubo anc in the general system, during the administration of plague serum. Naturally, in a case of bubonic plague the interest centres in the bubo. It is not proposed, however, to discuss the tissue changes met with in the bubo or organs generally, but attention will be exclusively directed to the changes undergone by the bacilli themselves in various parts of the body. As a rule, the first positive evidence of plague is obtained by puncture of the enlarged gland. The results so obtained may be briefly summarised as follows. In all cases examined during the first week of illness the existence of the bacillus pestis was readily demonstrated in the fluid aspirated from the bubo. It is a rather remarkable tact, and quite contrary to what might have been expected, that in all preparations so obtained the organisms, while presenting the characteristic appearances of the bacillus pestis, were present in comparatively small numbers even in the most severe cases. About the beginning of the second week the bacilli had mostly disappeared from the bubo, especially in the milder cases, and could only exceptionally be demonstrated after the second week, though in two cases they were found as late as the eighteenth day of illness. In no case was the bacillus recovered after this date. Although cultures made during this period remained sterile the microscope revealed the presence of sundry aberrant and obviously degenerated forms of the bacillus pestis. Generally, however, in those cases where the bubo had ruptured spontaneously the bacillus could not be demonstrated. In one case, although microscopical examination did not yield undoubted evidence of the presence of the bacillus, yet animal inoculation conclusively proved their existence in a state of undiminished virulence. Such an occurrence points to the unreliability of microscopical examination as an exclusive means of determining the period at which a patient may be declared non-infective. It was whilst observing the course of changes which occurred in the bubo in one particular case that attention was drawn to a peculiar alteration in the appearance of the bacilli in this situation after the administration of curative serum. These were found in a degenerated condition and that at a much earlier period than usual. The case referred to was that of a young female who had a large inguinal bubo which, when punctured on the second day of illness, yielded the characteristic bacillus on direct microscopical examination. On the third day of illness, however, the patient received an injection of 40 cubic centimetres of Yersin's serum in the lymphatic drain of the glands forming the bubo. The patient died early on the fourth day and post-mortem examination of the bubo showed a marked change in the characteristics of the bacilli present in this situation. They stained with difficulty and the usual bipolar appearance was lost owing to the stain being absorbed uniformly. Their outline also became indefinite and considerable variety of form was noted. The natural inference, therefore, was that the unusually early appearance of this degeneration was due, in all probability, to the local action of the serum. This conclusion was strengthened by the fact that the bacilli in the spleen preserted a perfec.ly 
normal appearance, a fact suggesting that while the amount of serum given was sufficient to affect the organisms in the bubo it was too small to influence the bacilli throughout the body generally. In this connexion it must be remembered, however, that the serum causes no such alteration "in vitro." This local effect of the serum on the bacilli in the inguinal bubo is shown in the accompanying illustration (Fig. 1), where the coccoid

FIG. 1.

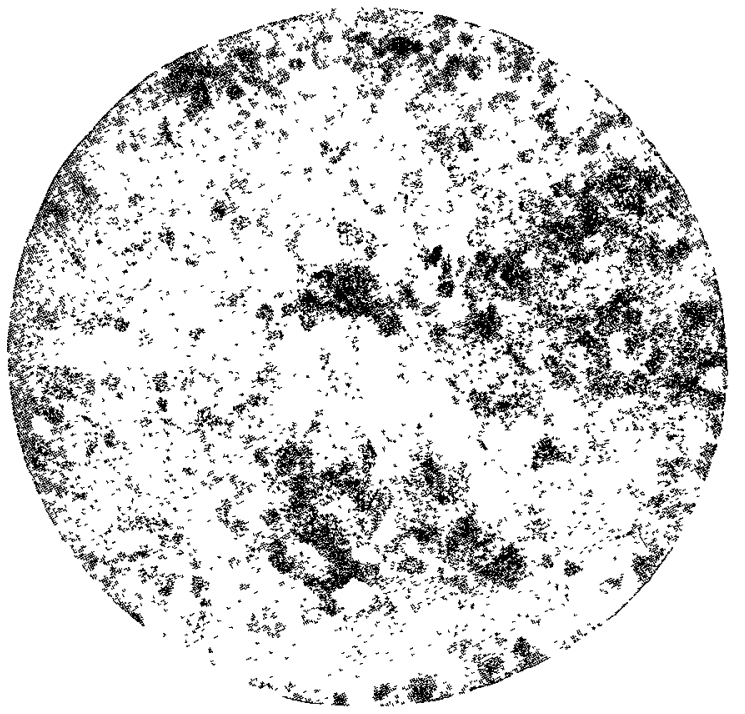

Degenerated plague bacilli from inguinal bubo after subcutaneous administration of serum. Fourth day of illness. $\times 1000$.

degeneration, the indefinite outline of the organisms, and the total loss of bipolar staining are very apparent. These appearances may be contrasted with those shown in Fig. 2,

FIG. 2.

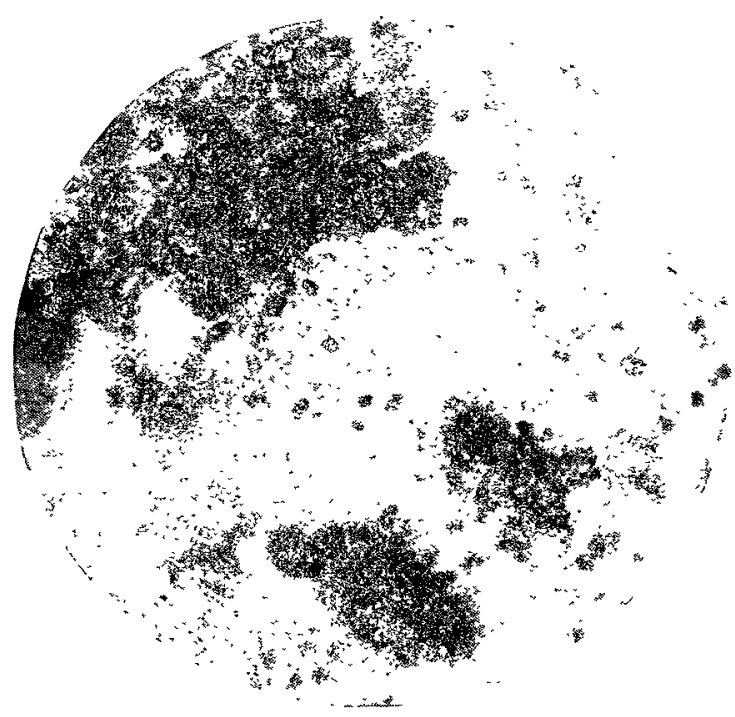

Showing characteristically stained plague bacilli in spleen of same case. Fourth day of illness. $\times 1000$

where the characteristic bipolar staining :of the bacilli present in the spleen is very evident.

As in some measure tending to confirm the view that those changes were due to the serum administered another fatal case of plague, in which curative serum was given both subcutaneously into the drain of the affected glands and also intravenously, may be cited. This patient was admitted to hospital on the fifth day of illness with a large and characteristic plague bubo in the right inguinal region. She was so seriously ill that no hope was entertained of her recovery, but 80 cubic centimetres of Yersin's serum were given subcutaneously by Dr. J. Brownlee into the inner aspect of the affected thigh. The following day she appeared much worse and 100 cubic centimetres were again given subcutaneously into the abdominal wall and 40 cubic centimetres intravenously. The same evening the patient died suddenly from acute heart failure. If the degenerative changes just described were the result of the local action of the serum on the organisms in the bubo it might reasonably be expected that similar changes would be found in the organisms present in the spleen and other tissues if the serum were administered intravenously. These conditions were actually found in the case just described and in the accompanying illustrations (Figs. 4 and 5) the changes in the morphological characters of the bacilli after the combined subcutaneous and intravenous administration of serum are clearly shown.

FIG. 3.

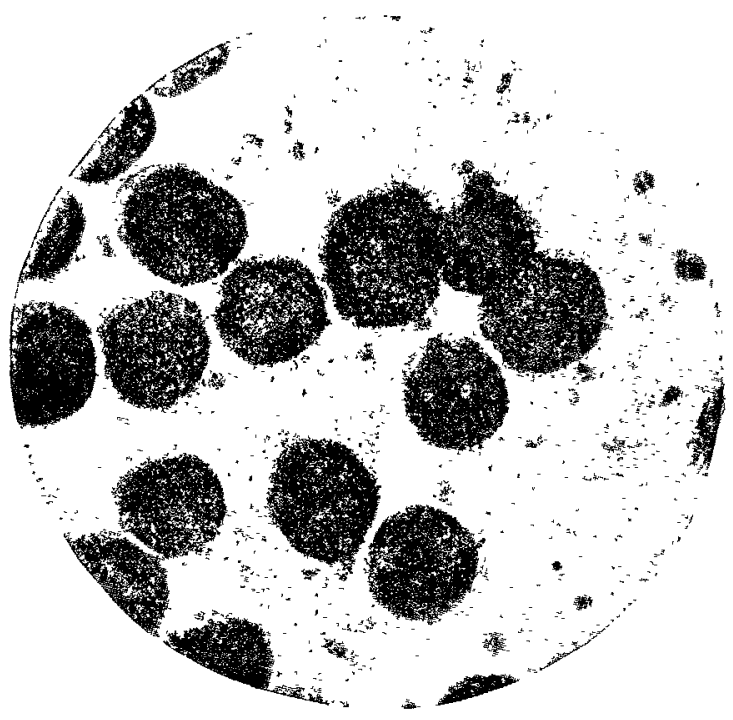

Bacillus pestis from bubo prior to administration of serum. Shows well-marked polar staining. Gentian violet, 2 $\times 1000$.

From an examination of these two cases it became apparent that a hitherto unnoticed mode of action of plague curative serum was evidently at work-viz., a directly bactericidal effect. In any disease of bacterial origin the effects produced-as evidenced by the symptoms of the disease-are generally ascribed not so much to the presence of the bacteria themselves (except in those instances where

FIG. 4.

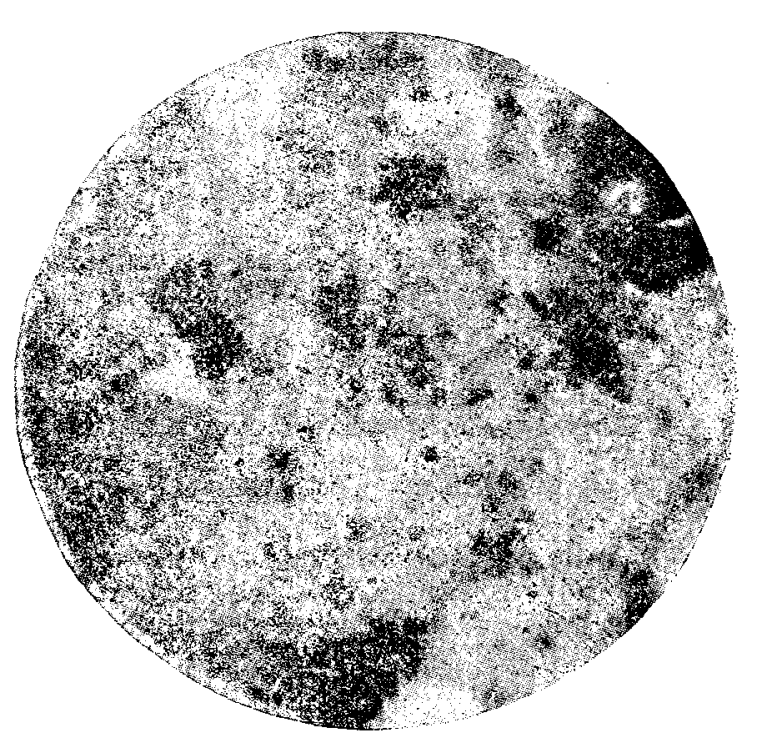

Bacillus pestis in bubo after subcutaneous administration of serum. Shows coccus-like degeneration of bacilli. Many of the more degenerated unstained shadowy forms are not visible. Gentian violet, $\times 1000$

their rapid multiplication mechanically obstructs the blood. vessels-e.g., anthrax) as to the specific effect of the toxin formed by them. To combat such a disease the remedy employed has to perform a double function: (1) the toxin

2 This illustration, as also the preceding (Fig. 2), gives a very deceptive impression of the number and appearance of bipolar.staining glass was in reality very considerable. 
already existing has to be neutralised, and (2) the bacteria have to be destroyed or their growth inhibited so that the further production of toxin may be lessened or arrested. The more or less considerable improvement in symptoms which has been found to follow the administration of Yersin's serum is in itself sufficient proof of its powerful antitoxic effects. From the microscopical observations just detailed,

FIG. 5.

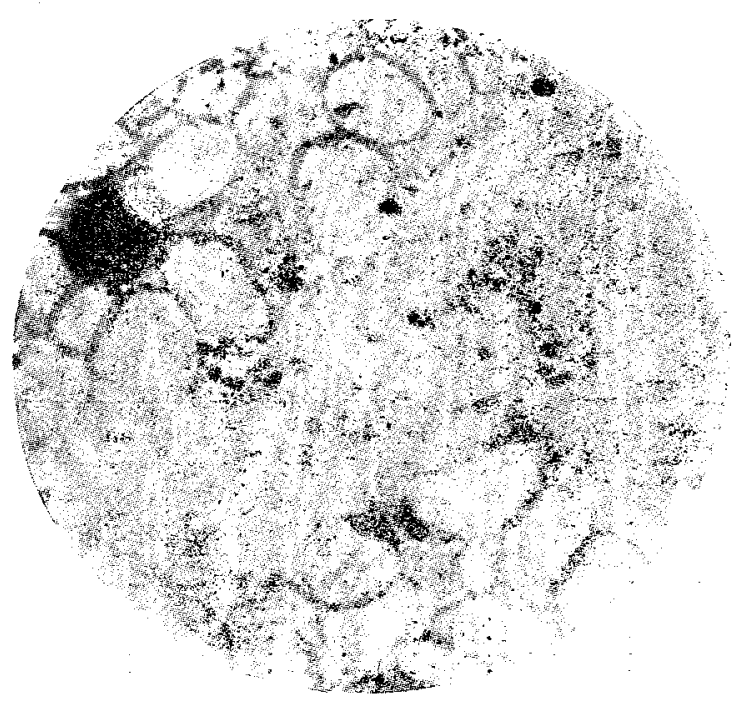

Bacillus pestis in spleen o: the same case after intravenous injection of serum. Degenerative changes are not so marked and a few normally stained bacilli are visible. Compare with Fig. 4. The comparative opacity in this field, as in the preceding, is rue to the enormous number of degenerated bacilli which fail to react towards the staining reagent. $\times 1000$.

CHART 1.

\begin{tabular}{|c|c|c|c|c|c|c|c|c|c|}
\hline DATE. & 14 & 15 & 16 & 'y & 18 & 19 & 20 & 21 & 22 \\
\hline $\begin{array}{l}\text { DAY OF } \\
\text { HLLNESS }\end{array}$ & $\begin{array}{c}2 \\
\mathrm{yE}\end{array}$ & $\begin{array}{c}3 \\
\square E\end{array}$ & $\begin{array}{c}4 \\
68\end{array}$ & $\sigma$ & 6 & $y_{E}$ & 8 & 9 & $\begin{array}{l}10 \\
4 \mathrm{E}\end{array}$ \\
\hline
\end{tabular}

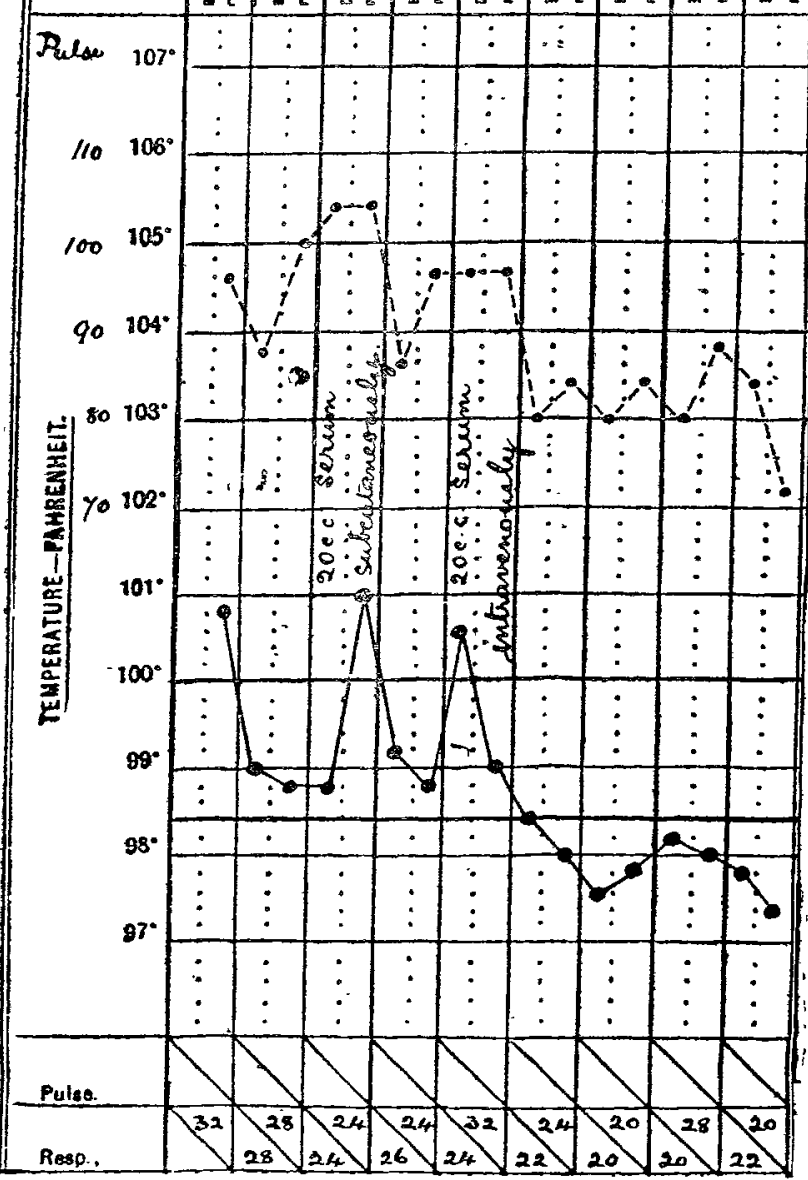

however, we have anatomical evidence of the bactericidal properties of the remedy. The serum apparently induces a degeneration in the bacilli themselves and these marked structural changes are bound to be associated with a considerable interference with their toxin-producing power. The remedy, therefore, strikes directly at the proximate cause of the disease-the bacilli-and also neutralises the toxins which have been produced. The evidence so obtained from microscopical examination, not only encouraged the freer exhibition of the remedy, but also gave a clue to the method which was ultimately found to yield the most beneficial results. Adequate doses had first to be administered locally in the drain of the bubo, so that the maximum bacillary degeneration might be speedily effected and the production of toxim arrested, and further, in order to reach those bacilli which might have escaped to the spleen or other organs and also to neutralise the toxin already existing in the blood the intravenous method of injection was indicated. Anatomically, it has just been shown that the serum has a local specific action on the bacilli in the bubo and that when introduced into the general circulation a like change was observed in those bacilli which had become lodged in the viscera. The microscopical evidence of bacillary degeneration in the spleen (shown in Fig. 5) encouraged one in the belief that larger doses of the serum intravenously would produce a still more decided change in these bacilli. Subsequent trial of this method was followed by clinical results which seemed to justify this assumption, but as all the cases so treated fortunately recovered no bacterioscopic information could be obtained of bacillary degeneration in deep organs. At this stage it may be of interest to recall briefly some of the cases in the earlier outbreak of which the following are the charts (Charts 1 and 2).

CHART 2.

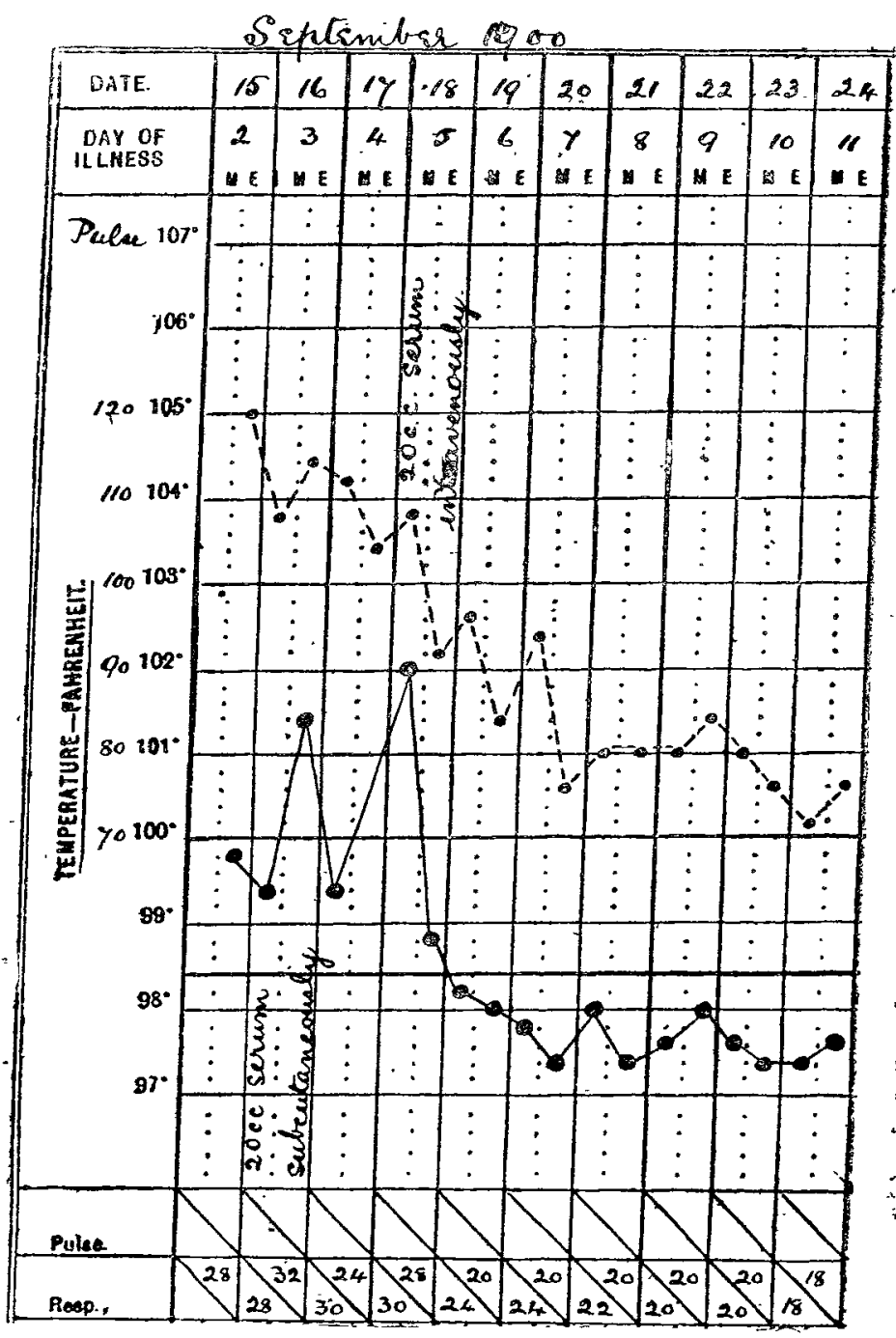

3 Report on certain cases of plague occurring in Glasgow in 1900, by the medical officer of health, pp. 49 and 51. 
It will be observed that after the subcutaneous injection improvement, as evidenced by fall in temperature and pulse, set in, though this was of temporary duration. On the other hand, when the intravenous method was employed the improvement was more decided and permanent. At the time when these cases were under observation no experience of the serum treatment of plague had been acquired and the remedy was used in a somewhat tentative manner until familiarity with its effects led to its freer exhibition. The cases just cited, though of a mild type, were proved to be genuine cases of plague, not only on clivical grounds but also by a complete chain of bacteriological evidence. It will be noted, however, that the serum was given in comparatively small doses. In the second outbreak of plague even more severe cases than were met with in the first epidemic were treated with comparatively large doses of serum, subcutaneously and intravenously, the experience already gained having encouraged this practice. The results way. I had already seen this patient about two or three weeks previously for a suspected gastric ulcer with purely gastric symptoms, and the pain on the present occasion being referred principally to the abdomen I at first thought that the present illness might be a return of the former, but on taking her temperature it was $106 \cdot 6^{\circ} \mathrm{F}$. and her pulse was running close on 140. As she was in extreme pain I gave her one-third of a grain of morphia hypodermically and stayed for some time to watch the case. I then made a more systematic examination of her and found a generalised scarlet rash on the trunk and also to some extent on the limbs. On palpation there was no special pain in the abdomen but in the right groin below Poupart's ligament was an exquisitely painful glandular swelling of about the size of a filbert. There were no swelling of the lymphatic vessels in the leg and no injury or abrasion of the limb. She had a slight sore throat of no great severity and without spots and there was no enlarge-

\section{CHART 3.}

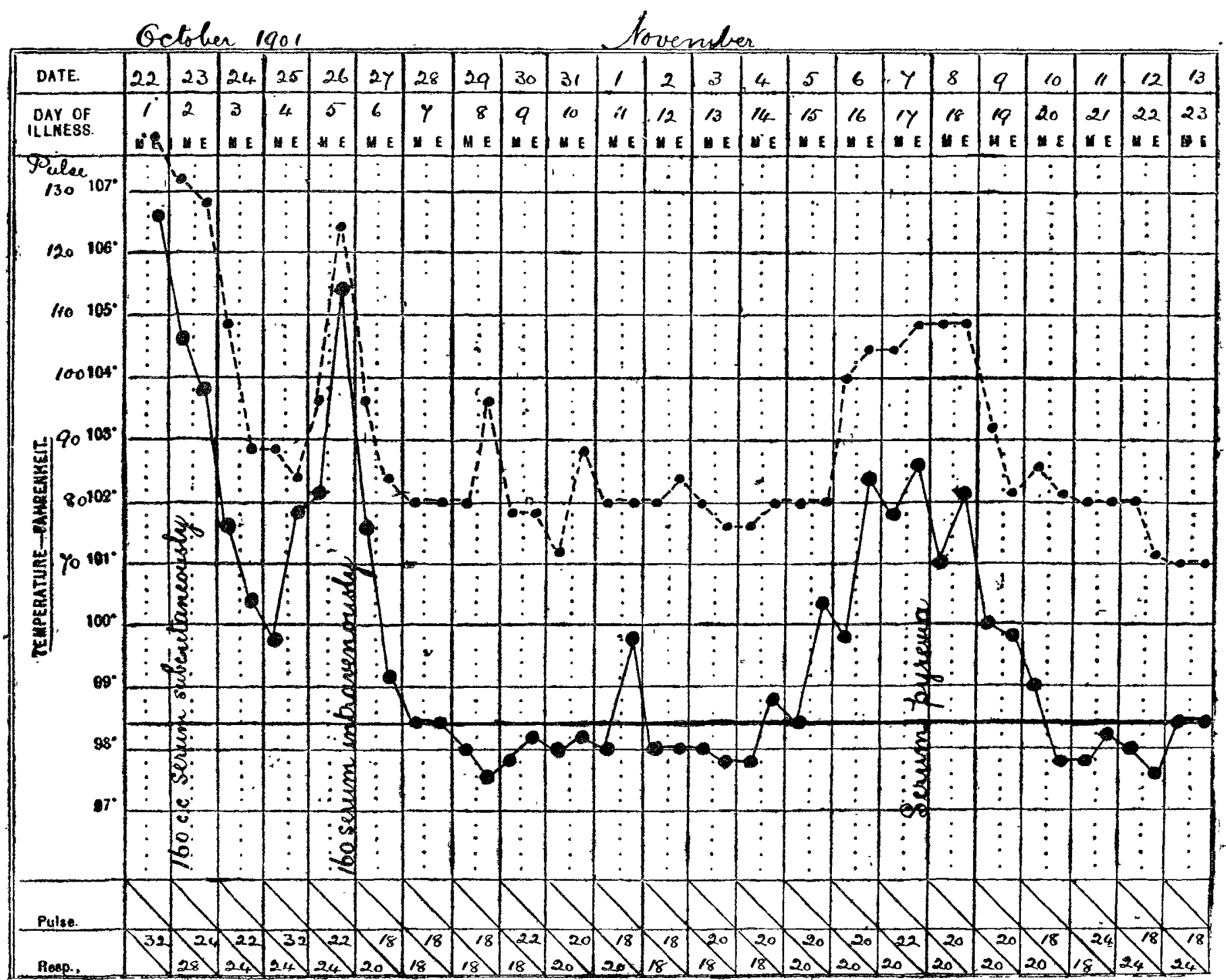

Temperature Chart of Case 1.

were eminently gratifying, as several exceptionally severe cases recovered under this more energetic line of treatment, but, of course, in these fortunate circumstances no evidence could be obtained of degeneration indaced in racilli throughout the body. The following two cases from the second outbreak are selected as being of an exceptionally severe type.

CASt 1.-A woman, aged 23 years, was admitted to hospital on Oct. 23rd, 1901. She was first seen by Dr. J. F. Fergus and the following is his note on the condition of the patient prior to her admission. "About 11.30 on the night of Tuesday, Oct. 22nd, I was called to see a young lady, an employée in a hotel in the city. I found the patient violently ill, complaining of severe pain in head and body, vomiting slightly and very much $f$ ushed, the face being markedly so, and the conjunctivæ suffused. She seemed seriously ill and answered my questions in a curious, slow, scanning sort of ment of the cervical glands or of those in the axilla. The clinical features of this case seemed to me to present an almost typical picture of the classical description of bubonic plague and before leaving the hotel I arranged for her immediate removal to Belvidere Fever Hospital. My suspicions had been particularly aroused by the assistant housekeeper mentioning casually that the patient had been complaining of a smell in the dessert-room where she worked and on my making more particular inquiries I learned that rats had been dying throughout the hotel in greater numbers than usual."

When admitted to hospital the patient was evidently acutely ill; the face was deeply flushed, the eyes were suffuied-in fact, congested-and the pupils were widely iilated. The tongue was moist and clean and showed no enlargement of the filiform papillæ. The fauces were slightly congested. The skin was hot and dry and both the trunk and the limbs were covered with a uniformly bright 
erythematous rash which momentarily faded under pressure, showing the skin to be perfectly pale and devoid of any of the usual yellow staining commonly seen in scarlet fever. The expression of the face was dull and heary yet anxious and the patient, though able to answer questions more or less intelligently, was evidently only half-conscious of her surroundings, for it was subsequently ascertained that she had no recollection of the bubo being punctured or of the first administration of serum. The condition of the bubo was as described in Dr. Fergus's note and so exquisitely tender that the slightest and gentlest manipulation was sufficient to rouse the patient from her lethargic, semi-comatose condition. Shortly after admission some blood was withdrawn from the bubo by means of a sterilised hypodermic needle and from this cover-glass films were made and glycerine agar tubes were inoculated. Immediate examination of the films, after staining with gentian violet, showed the presence, though in by a very rapid and striking amelioration in both the local and general condition of the patient, improvement setting in within six hours after the serum injection. The temperature abruptly fell from $106 \cdot 6^{\circ}$ to $100 \cdot 4^{\circ}$ the following evening and with this was associated a corresponding reduction in the pulse-rate from 132 to 88 . The patient was now perfectly conscious, the severe headache had entirely disappeared, and she spontaneously expressed herself as feeling much better. These favourable symptoms were associated with equally satisfactory changes in the bubo, the pain and teuderness of which were so much alleviated that it could be freely palpated without giving rise to any discomfort. This improvement, however, was shortlived, for on the next evening the temperature rose to $101 \cdot 8^{\circ}$ and during the succeeding 24 hours it continued to rise till at six o'clock on the evening of the 26 th (the sixth day of the illness) it stood at $105 \cdot 4^{\circ}$. The pulse also

CHART 4.

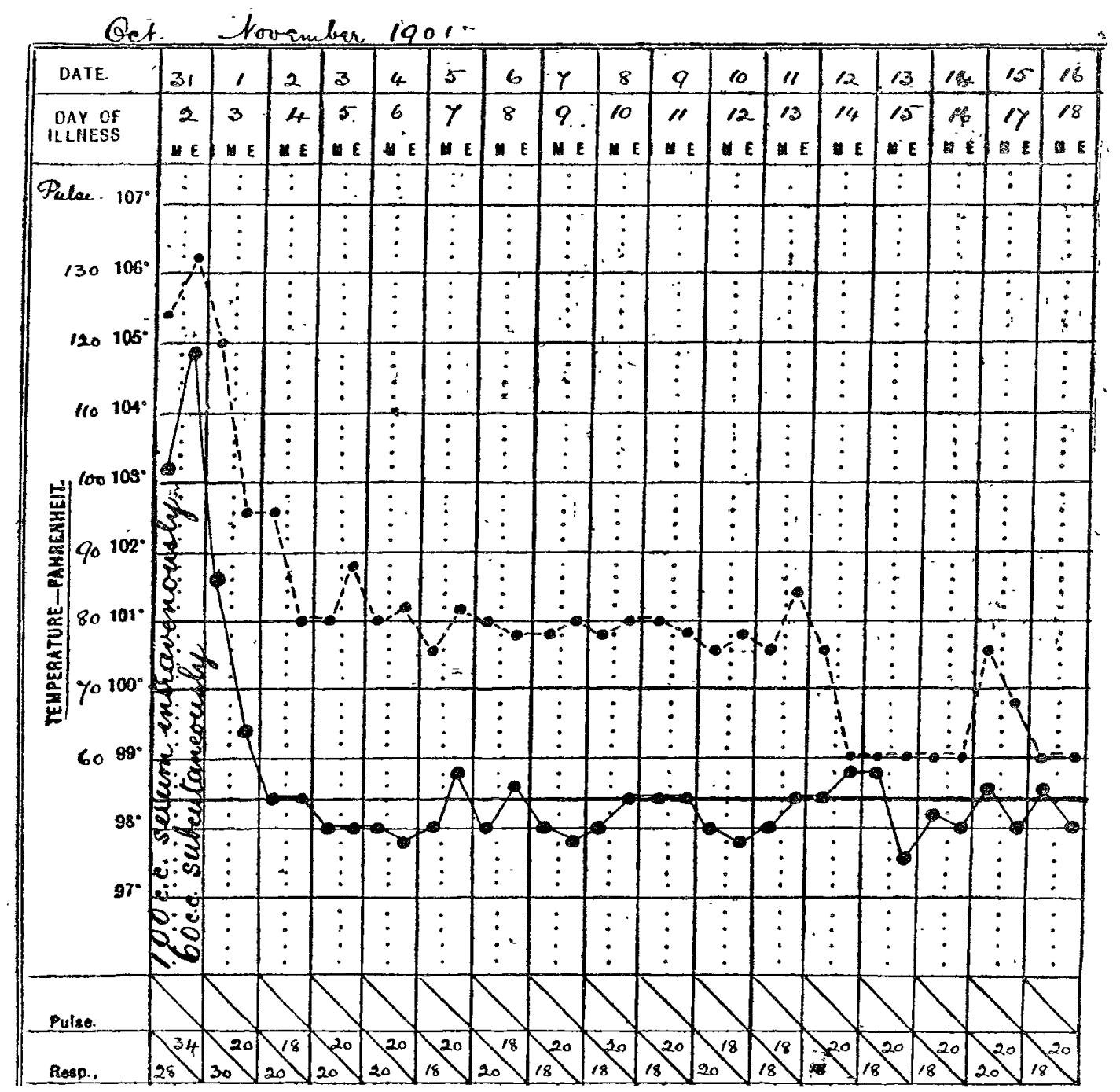

Temperature Chart of Case 2.

comparatively small numbers, of a short bipolar-staining bacillus morphologically identical with that of plague. When treated by Gram's method the bacillus was decolourised. The inoculated tub ss were incubated at $37^{\circ} \mathrm{C}$ and 36 hours later a faint white surface growth had appeared composed of small translucent colonies with serpiginous margins. Microscopical examination of cover-glass preparations made from these colonies showed a bacillus morphologically identical with, and presenting similar staining reactions to, those already described. 'The appearance of the cultures and stained films placed the diagnosis of the organism as bacillus pestis practically beyond a doubt. 160 cubic centimetres of Yersin's serum were administered subcutaneously on the same afternoon, the seat of injection being the thigh and in such a position that the serum would be absorbed by the lymphatics draining towards the affected glands. This was immediately followed was correspondingly rapid and more compressible than formerly. The bubo had increased somewhat in size and had again become acutely tenler, whilst her general condition reverted to that on admission. At this stage 160 cubic centimetres of serum were injected intravenously into the median basilic vein, and this was happily attended by even more gratifying results than those witnessed after the first administration of serum. Within 36 hours the temperature had fallen by a rapid crisis from $105.4^{\circ}$ to normal and with the exception of a trifling rise on Nov. 1st the latter figure was never exceeded during the following week. Defervescence was accompanied by a rapid and complete disappearance of all the threatening symptoms, by first a lessening and then by a complete cessation of pain in the bubo, and by a rapid return to consciousness and feeling of well-being. During the next few days the bubo enlarged somewhat and became highly inflamed though not at all 
painful. By the end of the second week it was quite luctuant and in appearance resembled an acute abscess. Spontaneous resolution, however, occurred without external rupture. Convalescence was rapid and complete and with the exception of some sharp febrile disturbance during the third week - due to severe articular pains consequent upon the administration of the serum-it was uneventful. The patient was dismissed on Dec. 12th and at this date the bubo was represented by a small horizontal chain of enlarged and indurated glands, quite devoid of pain and more or less firmly adherent to the skin and subjacent tissues.

To meet the extreme urgency of the case it was decided to administer a large dose of the serum subcutaneously ( 160 cubic centimetres as compared with from 20 to 60 cubic centimetres in former cases). So far as the immediate result was concerned the treatment seemed to be successful, but the improvement was short-lived, for, as has already been noted in the mild cases, the temperature quickly rose again and the patient's condition became almost as bad as before. The local symptoms having subsided somewhat, but the general condition of the patient pointing to increased action of the toxins on the system, it was considered advisable to administer more serum intravenously. 160 cubic centimetres were accordingly introduced into the median basilic vein. Almost immediately thereafter a marked improvement occurred which continued until nine days later when the usual serum pyrexia made its appearance (Chart 3 ).

The second case was also of a very severe type and occurred in association with the preceding.

CASE 2.-A woman, aged 33 years, was admitted to hospital on Oct. 31st, 1901. The patient had been "out of sorts" for a few days prior to the actual commencement of her illness, but the onset of severe symptoms occurred quite suddenly on the morning of Oct. 30th. She then became ill with violent headache, sickness, and vomiting, and pain in the left groin. All these symptoms rapidly increased in severity during the next 24 hours and on the following morning she was admitted to hospital in a profoundly prostrated condition. As in many respects the case was precisely similar to the preceding it is perhaps unnecessary to enter more fully into the details of the illness. The constitutional disturbance was considerable, the temperature being $104.8^{\circ} \mathrm{F}$. and the pulse being 124 . A relatively small but exquisitely painful bubo was present in the left groin about one and a half inches external to the spine of the pubes. The glands affected were those of the horizontal inguinal chain and so sensitive were they that the slightest pressure caused the most acute agony. On account of the gravity of the illness it was decided to at once administer serum by the combined method. 60 cubic centimetres were accordingly introduced into the lymphatic drain of the bubo and 100 cubic centimetres were introduced under hydrostatic pressure by means of a transfusion cannula into the median basilic vein. The reasons for administering serum both subcutaneously and intravenously have already been discussed and a reference to Chart 4 is sufficient to indicate the beneficial results which followed upon this line of treatment. Within 36 hours the temperature fell from $104 \cdot 8^{\circ}$ to normal and subsequent records never exceeded $98.8^{\circ}$. The pulse-rate was likewise lessened by 50 beats per minute. As in the previous case, a striking diminution in the tenderness of the bubo occurred a $f \in \mathbb{w}$ hours after injection, its further development appeared to be arrested, and spontaneous resolution occurred without rupture and without any apparent softening or inflammatory reaction. Convalescence was rapid and complete and when the patient was dismissed on Dec. 12th, 1901, the site of the bubo was indicated merely by two small indurated but perfectly painless glands about one inch outside the pubic spine.

Profiting by the experience already gained in the previous case serum was at once aciministered by the combined method. It was hoped by this plan to arrest the further production of the virus locally in the bubo and also to completely neutralise the toxin circulating in the blood. Both of these expectations were fulfilled, as was shown by the rapid and permanent improvement which took place locally and generally. The theory held regarding the previous case was that the primary fall of temperature depended largely upon the destructive action of the serum on the bacilli in the bubo and the neutralisation of the toxin in its immediate vicinity. The secondary rise, on the other hand, was believed to be due to the toxin already circulating or present in the various organs-e.g., the spleen, to which some bacilli might have gained access, and which, having escaped the local action of the serum given subcutaneously, continued to exert unimpaired their toxin-producing power. It must be admitted that the number of cases is too small to warrant general deductions, yet the experience gained is at least sufficient to suggest a more extended trial of what, in our limited experience, seems to be the most efficient method of administering plague serum. Beginning with the milder cases we find temporary improvement following upon subcutaneous injection, the final improvement depending upon the subsequent introduction of the remedy intravenously. Finally, it becomes evident from due consideration of the degree and nature of the improvement effected by the different methods that in severe cases the most satisfactory results are obtained by the simultaneous administration in adequate doses of subcutaneous and intravenous injections of Yersin's serum. This method, as is well known, was first suggested and employed by Calmette and Salimbeni during the Oporto epidemic in 1899."

Conclusions. -The experience gained during the two recent outbreaks of plague in this city seems to warrant the following conclusions: (1) that Yersin's serum is a remedy of the greatest value in the treatment of bubonic plague; (2) that its action is bactericidal, as shown by the degeneration in duced in the bacilli, as well as antitoxic ; (3) that this double action of the serum is best secured by its early administration in large doses, both subcutaneously into the lymphatic area which drains towards the bubo, and also intravenously; and (4) in very mild cases subcutaneous injection alone will probably suffice, but in severe cases the combined method should be employed. For these latter the initial combined dose should be perhaps from 150 to 300 cubic centimetres, the proportion given intravenously varying with the relative severity of the general symptoms.

Glasgow.

\section{A CASE OF TRANS-SACRAL REMOVAL OF AN INTUSSUSCEPTION WITH MALIGNANT GROWTH BY A METHOD UNUSUAL AT PRESENT.}

BY ARTHUR E. BARKER, F.R.C.S. ENG.,

SURGEOX TO UXIVERSITY COLLEGE HOSPITAL, ETC.

A BRIEF record of the following case may, I venture to think, prove useful to those who like myself are much exercised in their minds as to the proper course to take in regard to the treatment of carcinoma of the rectum. On the one hand we have to decide whether simply to palliate by colotomy or on the other whether a more radical operation for the removal of the growth is called for. And if we answer the last question in the affirmative we have to settle in our minds which of many procedures holds out the best prospect of permanent success. The pathological condition, too, met with in this case has many points of interest.

A single woman, aged 52 years, was sent to me on April $29 \mathrm{th}, 1902$, by Mr. F. Hart-Smith of Leominster with the following history. She was reported to have had for a couple of weeks before being seen a discharge of blood and mucus from the bowel without obstruction, distension, or pain. On examination her medical man felt an ulcerated mass in the rectum which he took to be carcinoma, in which, as it turned out, he was quite correct. I was surprised, therefore, when I first examined the rectum to find nothing abnormal with my finger, although the latter came away covered with blood-stained mucus. I then introduced a long glass Ferguson's speculum and examined with a good electric light. But nowhere could I see anything abnormal except some blood and mucus which came from high up. Nevertheless, I felt sure that my old friend's diagnosis was correct and that $I$ was unable to find what he described because it had slipped up. I took the condition to be one of a carcinomatous ring causing an intussusception which had become reduced. This, although not very common, is a condition most likely familiar to many surgeons and one on which I have several times operated. I wrote in this

4 La Peste Bubonique, par Calmette et Salimbeni, Annales de l'Institut Pasteur, Decembre, 1899, p. 899. 